\title{
Wave Mode Control of Cantilever Slab Structure of T-Beam Bridge with Large Aspect Ratio
}

\author{
Tingting Liu $(\mathbb{D}$, Chuanping Zhou $(\mathbb{D}$, Zhigang Yan, and Guojin Chen \\ School of Mechanical Engineering, Hangzhou Dianzi University, Hangzhou 310018, China \\ Correspondence should be addressed to Chuanping Zhou; zhoucp@hdu.edu.cn
}

Received 15 November 2021; Accepted 16 December 2021; Published 30 December 2021

Academic Editor: Nan Zhang

Copyright ( $\odot 2021$ Tingting Liu et al. This is an open access article distributed under the Creative Commons Attribution License, which permits unrestricted use, distribution, and reproduction in any medium, provided the original work is properly cited.

\begin{abstract}
The cantilever plate structure in a T-beam bridge with a large aspect ratio will cause vibration under the influence of environmental disturbance and self-stress, resulting in fatigue damage of the plate structure. Wave control based on elastic wave theory is an effective method to suppress the vibration of the cantilever plate structure in a beam bridge. Based on the classical thin plate theory and the wave control method, the active vibration control of the T-shaped cantilever plate with a large aspect ratio in the beam bridge is studied in this paper. The wave mode control strategy of structural vibration is analyzed and studied, the controller is designed, the vibration mode function of the cantilever plate is established, and the control force/sensor feedback wave control is implemented for the structure. The dynamic response of the cantilever plate before and after applying wave control force is analyzed through numerical examples. The results show that the response of the structure is intense before control, but after wave control, the structure increases damping, absorbs the energy carried by the elastic wave in the structure, weakens the sharp response, and changes the natural frequency of the structure to a certain extent.
\end{abstract}

\section{Introduction}

The bridge structure in working condition will bear its static load and dynamic load caused by peripheral vibration. There are a large number of T-cantilever extended plate structures in beam bridge structures, mostly $\mathrm{T}$-cantilever plate structures with a large aspect ratio. These structures carry their static load and the static load and dynamic load of surrounding vehicles in the beam bridge. At the same time, the environmental disturbance and dynamic rotation of the beam bridge structure will also cause structural vibration $[1,2]$. The abovementioned will cause the vibration of the bridge structure. If effective vibration suppression measures are not taken, the vibration will decay very slowly, which will not only affect the position of the beam bridge structure, but also cause the vibration of the plate structure, cause fatigue damage, and have a serious impact on the safety and service life of the beam bridge [3].

The traditional passive control methods, such as adding damping, often only increase the additional weight of the structure and reduce the service efficiency of the plate structure and cannot get satisfactory results [4]. Therefore, wave control based on elastic wave theory to suppress the vibration of the cantilever plate structure in the beam bridge is a new method to solve this problem.

At present, in the research of wave control, the research object is mostly simplified as a flexible beam, and some literature are simplified as plate structure to implement the bending wave control $[5,6]$. The wave control method can be used to control the propagation of waves in one-dimensional structures, such as bending waves in beams, and axial waves in rods.

Halkyard studied the feedback adaptive control of bending vibration of beam structure by the wave control method [7]. EL-Khatib employs a tuned vibration absorber to investigate the bending wave suppression in beams [8]. Krushynska studied the propagation characteristics of curved edge waves in semi-infinite isotropic elastic plates and found that the velocity of edge waves is essentially independent of Poisson's ratio [9]. Jones, IS used the same method to analyze and experiment with the active control of semi-infinite simply supported rib [10]. Kaplunov studied 
the propagation of a three-dimensional edge wave along the edge of a semi-infinite elastic plate under mixed boundary conditions and found that the cut-off frequency of the edge wave is consistent with the natural frequency of the semiinfinite strip [11]. In this paper, based on the research of the abovementioned documents, a large number of cantilever plate structures existing in the girder bridge structure are controlled by the sliding film variable structure control method which is different from the conventional control method.

The vibration of the structure can be regarded as the superposition of the waves propagating in the structure, which are reflected and transmitted at the discontinuity of the structure [12, 13]. By controlling the transmitted or absorbed wave energy from one side to the other side of the structure, the structural vibration can be suppressed.

Based on the classical thin plate theory and the wave control method, the active vibration control of T-cantilever plate with a large aspect ratio in beam bridge is studied in this paper. The wave mode control strategy of structural vibration is analyzed and studied. The controller is designed to control the force/sensor feedback wave of the structure.

\section{Vibration Equation and Solution of Thin Plate}

According to the classical thin plate theory, the expression of displacement component $u v$ in the rectangular coordinate system is $[14,15]$

$$
\begin{aligned}
& u(x, y, z, t)=-z \frac{\partial w(x, y, t)}{\partial x}, \\
& v(x, y, z, t)=-z \frac{\partial w(x, y, t)}{\partial y},
\end{aligned}
$$

where $w(x, y, t)$ represents the transverse displacement of the plate, $u(x, y, z, t)$ and $v(x, y, z, t)$ represent the displacement in $x$ and $y$ directions in the plane, respectively, which is linearly distributed along the thickness direction.

The bending moment and shear force in the plate can be described as follows:

$$
\begin{aligned}
M_{x} & =-D\left(\frac{\partial^{2} w}{\partial x^{2}}+v \frac{\partial^{2} w}{\partial y^{2}}\right), \\
M_{y} & =-D\left(\frac{\partial^{2} w}{\partial y^{2}}+v \frac{\partial^{2} w}{\partial x^{2}}\right), \\
M_{x y} & =M_{y x} \\
& =-D(1-v) \frac{\partial^{2} w}{\partial x \partial y}, \\
Q_{x} & =-D \frac{\partial}{\partial x} \nabla^{2} w \\
Q_{y} & =-D \frac{\partial}{\partial y} \nabla^{2} w
\end{aligned}
$$

where $D$ is the bending stiffness of the plate, $D=E h^{3} / 12\left(1-v^{2}\right), \quad \nabla^{2}=\partial^{2} / \partial x^{2}+\partial^{2} / \partial y^{2}$ is the Laplace operator, and $v$ is the Poisson's ratio.

The cantilever plate structure studied in this paper is shown in Figure 1. The equilibrium equation of plate microelement is

$$
\begin{array}{r}
\frac{\partial M_{x}}{\partial x}+\frac{\partial M_{y x}}{\partial y}-Q_{x}=0, \\
\frac{\partial M_{x y}}{\partial x}+\frac{\partial M_{y}}{\partial y}-Q_{y}=0, \\
\frac{\partial Q_{x}}{\partial x}+\frac{\partial Q_{y}}{\partial y}+\bar{q}-\rho h \frac{\partial^{2} w}{\partial t^{2}}=0,
\end{array}
$$

where $\rho$ is the mass of the plate per unit area and $\bar{q}$ is the external load on the plate per unit area.

Substituting equation $(2 \mathrm{a})$ in $(3 \mathrm{a})$, the vibration differential equation of the thin plate is

$$
\begin{array}{r}
\frac{\partial^{4} w}{\partial x^{4}}+2 \frac{\partial^{4} w}{\partial x^{2} \partial y^{2}}+\frac{\partial^{4} w}{\partial y^{4}}+\frac{\rho h}{D} \frac{\partial^{2} w}{\partial t^{2}}=\frac{\bar{q}(x, y, t)}{D}, \\
\nabla^{2} \nabla^{2} w+\frac{\rho h}{D} \frac{\partial^{2} w}{\partial t^{2}}=\frac{\bar{q}(x, y, t)}{D} .
\end{array}
$$

The displacement expression of modal expansion form of thin plate is

$$
w(x, y, t)=\sum_{i=1}^{m} \sum_{j=1}^{n} W_{i, j}(x, y) q_{i, j}(t),
$$

where $W_{i, j}(x, y)$ is the vector modal function, $q_{i, j}(t)$ is the modal coordinate, and $m, n$ are the modal truncation numbers.

Substitute equation (5) in (4a) to obtain

$$
\sum_{i=1}^{m} \sum_{j=1}^{n} \nabla^{4} W_{i, j} q_{i, j}+\sum_{i=1}^{m} \sum_{j=1}^{n} \frac{\rho h}{D} W_{i, j} \frac{\partial^{2} q_{i, j}}{\partial t^{2}}=\frac{\bar{q}(x, y, t)}{D}
$$

where $W_{i, j}(x, y)$ shall satisfy the following equation:

$$
\nabla^{4} W_{i, j}-\omega_{i, j}^{2} \frac{\rho h}{D} W_{i, j}=0
$$

where $\omega_{i . j}$ is the natural frequency of the plate.

Substitute equation (7) in (6) to obtain

$$
\sum_{i=1}^{m} \sum_{j=1}^{n}(\rho h) W_{i, j} \frac{\partial^{2} q_{i, j}}{\partial t^{2}}+\sum_{i=1}^{m} \sum_{j=1}^{n}(\rho h) \omega_{i, j}^{2} W_{i, j} q_{i, j}=\bar{q} .
$$

Multiply both ends of equation (8) by the vibration mode function $W_{r, s}(x, y)$, it is integrated on the area domain $\Omega$ of the thin plate, and the orthogonality of the structural vibration mode shape function is used. 


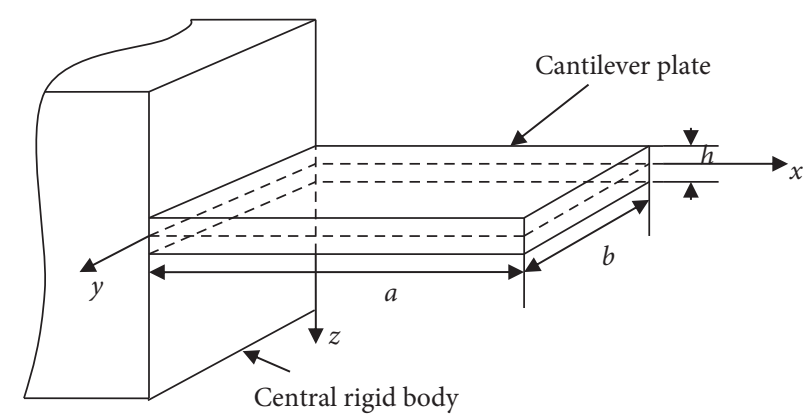

Figure 1: Model of the cantilever plate.

$$
\frac{1}{m_{i, j}} \iint_{\Omega} W_{i, j}(\rho h) W_{r, s} \mathrm{~d} x \mathrm{~d} y=\left\{\begin{array}{l}
0 \quad i \neq r, j \neq s \quad i, r=1,2 \ldots m j, s=1,2 \ldots n \\
1 \quad i=r, j=s \quad i, r=1,2 \ldots m j, s=1,2 \ldots n
\end{array}\right.
$$

The result is as follows:

$$
\iint_{\Omega} \sum_{i=1}^{m} \sum_{j=1}^{n} W_{i, j}(\rho h) W_{r, s} \frac{\partial^{2} q_{i, j}}{\partial t^{2}} \mathrm{~d} x \mathrm{~d} y+\iint_{\Omega} \sum_{i=1}^{m} \sum_{j=1}^{n} \omega_{i, j}^{2} W_{i, j}(\rho h) W_{r, s} q_{i, j} \mathrm{~d} x \mathrm{~d} y=\iint_{\Omega} W_{r, s} \bar{q}(x, y, t) \mathrm{d} x \mathrm{~d} y .
$$

Therefore, there are

$$
\frac{\partial^{2} q_{i, j}(t)}{\partial t^{2}}+\omega_{i, j}^{2} q_{i, j}(t)=p_{i, j}(t), \quad i=1,2, \ldots m ; j=1,2, \ldots n
$$

Among them, the external disturbing force $p_{i, j}(t)=1 / m_{i, j} \iint_{\Omega} \bar{q}(x, y, t) W_{i, j}(x) \mathrm{d} x \mathrm{~d} y$, equation (11) is the modal coordinate equation of the plate.

The lateral displacement of the points on the plate is expanded into the form of modal superposition. For cantilever rectangular plates, the modal function can usually be decomposed into the product of two beam functions [16], that is, the product of cantilever beam in $x$-direction and free beam at both ends in $y$-direction. Therefore, the deflection of the plate can be expressed as

$$
w(x, y, t)=\sum_{i=1}^{m} \sum_{j=1}^{n} W_{i}(x) W_{j}(y) q_{i, j}(t)
$$

where $W_{i}(x)$ is the mode function of the transverse displacement of the cantilever beam in the $x$-direction of the plate and $W_{j}(y)$ is the modal function of the free beam in the $y$-direction of the plate.

2.1. Vibration Mode Function of Cantilever Plate in $x$ Direction. Based on Euler Bernoulli beam theory, the $i$ order modal function of transverse displacement in $x$-direction can be expressed as

$$
W_{i}(x)=D_{1} e^{-i k_{0 i} x}+D_{2} e^{i k_{0 i} x}+D_{3} e^{-k_{0 i} x}+D_{4} e^{k_{0 i} x} .
$$

Applying Euler formula,

$$
\left.\begin{array}{l}
e^{ \pm i k x}=\cos (k x) \pm i \sin (k x) \\
e^{ \pm k x}=\cosh (k x) \pm \sinh (k x)
\end{array}\right\} .
$$

By substituting (14) in (13), another expression of the vibration mode function can be obtained.

$$
\begin{aligned}
W_{i}(x)= & A_{1} \cos \left(k_{0 i} x\right)+A_{2} \sin \left(k_{0 i} x\right) \\
& +A_{3} \cosh \left(k_{0 i} x\right)+A_{4} \sinh \left(k_{0 i} x\right) .
\end{aligned}
$$

Considering the boundary conditions at both ends of the cantilever Euler Bernoulli beam, it can be obtained that

$$
\cos \left(k_{0 i} a\right) \cdot \cosh \left(k_{0 i} a\right)+1=0 .
$$

Equation (16) is the dispersion equation of cantilever vibration [17].

Combined with the frequency equation, equation (15) can be expressed as

$$
\begin{aligned}
W_{i}(x)= & \cos h\left(k_{0 i} x\right)-\cos \left(k_{0 i} x\right) \\
& +a_{i}\left[\sin h\left(k_{0 i} x\right)-\sin \left(k_{0 i} x\right)\right],
\end{aligned}
$$

where $a$ is the length in the $x$-direction, $k_{0 i}=\left(\rho h \omega^{2} / D\right)^{1 / 4}$ is the elastic wave number in the classical plate; $a_{i}=-\cosh \left(k_{0 i} a\right)+\cos \left(k_{0 i} a\right) / \sinh \left(k_{0 i} a\right)+\sin \left(k_{0 i} a\right)$, this coefficient is determined according to the boundary conditions at both ends of the cantilever Euler Bernoulli beam. Equation (17) is the vibration mode function of the cantilever plate in the $x$-direction. 
2.2. Vibration Mode Function of Cantilever Plate in $y$-Direction. In the same way as the derivation of the mode shape function in the $x$-direction, the $j$-order mode shape function of the transverse displacement in the $y$-direction of the cantilever plate is

$$
W_{j}(y)=C_{1} e^{-i k_{0 j} y}+C_{2} e^{i k_{0 j} y}+C_{3} e^{-k_{0 j} y}+C_{4} e^{k_{0 j} y} .
$$

Using Euler formula (14), change $x$ into $y$ and substitute it in (18)

$$
\begin{aligned}
W_{j}(y)= & B_{1} \cos \left(k_{0 j} y\right)+B_{2} \sin \left(k_{0 j} y\right) \\
& +B_{3} \cosh \left(k_{0 j} y\right)+B_{4} \sin h\left(k_{0 j} y\right)
\end{aligned}
$$

Considering the boundary conditions at both ends of the Euler Bernoulli beam with free ends, it can be obtained that

$$
\cos \left(k_{0 j} b\right) \cdot \cosh \left(k_{0 j} b\right)-1=0 .
$$

Equation (20) is the dispersion equation of the vibration of free beams at both ends [18, 19].

Combined with the frequency equation, equation (20) can be expressed as

$$
\begin{aligned}
W_{j}(y)= & \cosh \left(k_{0 j} y\right)+\cos \left(k_{0 j} y\right) \\
& +b_{i}\left[\sinh \left(k_{0 j} y\right)+\sin \left(k_{0 j} y\right)\right],
\end{aligned}
$$

where $b$ is the length in the $y$-direction, $k_{0 j}=\left(\rho h \omega^{2} / D\right)^{1 / 4}$ is the elastic wave number in the classical plate; $b_{j}=-\cosh \left(k_{0 j} b\right)-\cos \left(k_{0 j} b\right) / \sinh \left(k_{0 j} b\right)-\sin \left(k_{0 j} b\right)$, this coefficient is determined according to the boundary conditions of Euler Bernoulli beams with free ends.

It should be emphasized here that when $j \geq 3$, the mode function of the Euler Bernoulli beam with free ends is equation (21), which is determined by the dispersion equation of vibration of free beams at both ends. And, $W_{j}(j=1,2)$ is in the following form:

$$
\begin{aligned}
& W_{1}=1, \\
& W_{2}=1-\frac{2 y}{b} .
\end{aligned}
$$

It can be seen that equations (21) and (22) are vibration mode functions in the $y$-direction of the cantilever plate.

The maximum kinetic energy of the plate during vibration is [20]

$$
T_{\max }=\frac{1}{2} \iint_{\Omega} \rho h W^{2}(x, y) \mathrm{d} x \mathrm{~d} y .
$$

The maximum potential energy of the plate during vibration is

$$
U_{\max }=\frac{1}{2} \iint_{\Omega} D\left\{\left(\frac{\partial^{2} W}{\partial x^{2}}+\frac{\partial^{2} W}{\partial y^{2}}\right)^{2}+2(1-v)\left[\left(\frac{\partial^{2} W}{\partial x \partial x}\right)^{2}-\frac{\partial^{2} W}{\partial x^{2}} \frac{\partial^{2} W}{\partial y^{2}}\right]\right\} \mathrm{d} x \mathrm{~d} y
$$

According to the Rayleigh-Ritz principle, the natural frequency of the plate is

$$
\omega^{2}=\frac{U_{\max }}{T_{\max }}
$$

\section{Feedback Wave Control of Cantilever Plate}

Consider the presence of an excitation in the beam structure where a train of wave control forces is applied to a line parallel to the $y$-axis. At this point, the point discontinuity on the beam is the line discontinuity here. According to the traveling wave theory, the incident elastic wave will be reflected and transmitted at discontinuities [21, 22]. Assuming a column of forward propagating waves is incident at $x=0$, where the incident attenuation wave is ignored and the time factor is not considered, the displacements of the beam at $x<0$ and $x>0$ are respectively as follows:

$$
\begin{aligned}
& w_{-}(x, y)=a^{+} e^{-i k x} W(y)+a^{-} e^{i k x} W(y)+a_{N}^{-} e^{k x} W(y), \\
& w_{+}(x, y)=b^{+} e^{-i k x} W(y)+b_{N}^{+} e^{-k x} W(y) .
\end{aligned}
$$

Make

$$
\begin{aligned}
& {\left[\begin{array}{l}
b^{+} \\
b_{N}^{+}
\end{array}\right]=\left[\begin{array}{l}
t_{1} \\
t_{2}
\end{array}\right] a^{+},} \\
& {\left[\begin{array}{l}
a^{-} \\
a_{N}^{-}
\end{array}\right]=\left[\begin{array}{l}
r_{1} \\
r_{2}
\end{array}\right] a^{+},}
\end{aligned}
$$

where $a^{+}$is the mode coefficient of the incident propagation wave, $a^{-}$is the mode coefficient of the reflected propagation wave, $a_{N}^{-}$is the mode coefficient of the reflected attenuation wave, $b^{+}$is the mode coefficient of the transmitted propagation wave, and $b_{N}^{+}$is the mode coefficient of the transmitted attenuation wave.

In feedback wave control, sensors and actuators are positioned in an area of the structure to control the propagation of elastic waves, where the position of the control force is discontinuous. In the frequency domain, the control force of the feedback wave is taken as

$$
F(\omega)=-H(\omega) W(\omega),
$$

where $H(\omega)$ is the transfer function of the controller, which can be determined by calculating the reflection coefficient and transmission coefficient of the solution wave. 
Considering the continuity and balance of the beam at $x=0$, there are

$$
\begin{aligned}
w_{+}(0, y) & =w_{-}(0, y), \\
\frac{\partial w_{-}(0, y)}{\partial x} & =\frac{\partial w_{+}(0, y)}{\partial x}, \\
H w_{+} & =Q_{+}(0, y)-Q_{-}(0, y), \\
M_{+}(0, y) & =M_{-}(0, y),
\end{aligned}
$$

where $H$ is the transfer function of the wave controller.

Substitute equation (26) in (29a)

$$
\begin{gathered}
{\left[\begin{array}{c}
1 \\
-i
\end{array}\right] a^{+}+\left[\begin{array}{ll}
1 & 1 \\
i & 1
\end{array}\right]\left[\begin{array}{l}
a^{-} \\
a_{N}^{-}
\end{array}\right]=\left[\begin{array}{cc}
1 & 1 \\
-i & -1
\end{array}\right]\left[\begin{array}{l}
b^{+} \\
b_{N}^{+}
\end{array}\right],} \\
{\left[\begin{array}{c}
i \\
-1
\end{array}\right] a^{+}+\left[\begin{array}{cc}
-i & 1 \\
-1 & 1
\end{array}\right]\left[\begin{array}{c}
a^{-} \\
a_{N}^{-}
\end{array}\right]=\left[\begin{array}{cc}
i & -1 \\
-1 & 1
\end{array}\right]\left[\begin{array}{c}
b^{+} \\
b_{N}^{+}
\end{array}\right]+\left[\begin{array}{cc}
\bar{H} & \bar{H} \\
0 & 0
\end{array}\right]\left[\begin{array}{c}
b^{+} \\
b_{N}^{+}
\end{array}\right],}
\end{gathered}
$$

where $\bar{H}=H / E I k^{3}$.

Substitute equation (27) in (30a),

$$
\begin{array}{r}
{\left[\begin{array}{c}
1 \\
-i
\end{array}\right] a^{+}+\left[\begin{array}{ll}
1 & 1 \\
i & 1
\end{array}\right]\left[\begin{array}{l}
r_{1} \\
r_{2}
\end{array}\right] a^{+}=\left[\begin{array}{cc}
1 & 1 \\
-i & -1
\end{array}\right]\left[\begin{array}{l}
t_{1} \\
t_{2}
\end{array}\right] a^{+},} \\
{\left[\begin{array}{c}
i \\
-1
\end{array}\right] a^{+}+\left[\begin{array}{cc}
-i & 1 \\
-1 & 1
\end{array}\right]\left[\begin{array}{l}
r_{1} \\
r_{2}
\end{array}\right] a^{+}=\left[\begin{array}{cc}
i & -1 \\
-1 & 1
\end{array}\right]\left[\begin{array}{l}
t_{1} \\
t_{2}
\end{array}\right] a^{+}+\left[\begin{array}{cc}
\bar{H} & \bar{H} \\
0 & 0
\end{array}\right]\left[\begin{array}{l}
t_{1} \\
t_{2}
\end{array}\right] a^{+} .} \\
g=\frac{\sqrt{2}}{\omega}
\end{array}
$$

By solving this equation

$$
\begin{aligned}
& t_{1}=1+\frac{\bar{H} i}{4-(1+i) \bar{H}}, \\
& r_{1}=\frac{\bar{H} i}{4-(1+i) \bar{H}} .
\end{aligned}
$$

The energy carried by the propagating wave is proportional to the square of the wave amplitude [23]. Therefore, the reflection energy and transmission energy per unit incident energy is $E=\left|t_{1}\right|^{2}+\left|r_{1}\right|^{2}$, if there is no energy dissipation at $x=0$, then $\left|t_{1}\right|^{2}+\left|r_{1}\right|^{2}=1$. In this paper, a controller for absorbing incident energy is designed by adding damping to the structure.

In particular, if we let $\bar{H}(\omega)=\sqrt{2}(1+i) \omega g$, then

$$
E=\left|t_{1}\right|^{2}+\left|r_{1}\right|^{2}=\left|1-\frac{(1+i) g \omega}{2 g \omega+2 \sqrt{2} i}\right|^{2}+\left|-\frac{(1+i) g \omega}{2 g \omega+2 \sqrt{2} i}\right|^{2} \text {. }
$$

We assume that the wave is incident from one side of the control region to seek the optimal control gain $g$ and make it absorb the energy of the incident wave as much as possible, in other words, minimize $E=|r|^{2}+|t|^{2}$ (maximize the energy absorbed by the controller). In this case, let $\partial E / \partial \omega=0$, and the control gain is
Therefore, the transfer function of the wave controller is

$$
\bar{H}(\omega)=2(1+i) .
$$

Typically, the tuned PD (proportional plus differential control) feedback wave control is used so that it has the same effect as the optimal controller at a particular frequency $\omega_{d}$. The frequency response of the controller is

$$
\bar{H}_{w}(\omega)=c_{1}+c_{2}(i \omega)
$$

where $c_{1}=2, c_{2}=2 / \omega_{d}$.

When the controller in the frequency domain is converted to the time domain, the inverse Fourier transform is performed on the control law, and the PD control in the time domain is obtained $[24,25]$. If the wave control force is applied at $\left(x_{w}, y_{w}\right)$, it becomes

$$
f_{w}(x, y, t)=-\left[c_{1} w(x, t)+c_{2} \dot{w}(x, t)\right] \delta\left(x-x_{w}\right) \delta\left(y-y_{w}\right) .
$$

At this time, the wave control force (37) is applied to the original vibration system, and the matrix form of the system motion equation is

$$
\tilde{M}_{w} \ddot{q}+\widetilde{C}_{w} \dot{q}+\widetilde{K}_{w} q=\tilde{p},
$$


where $\quad \tilde{p}=\left[\begin{array}{llll}p_{11} & p_{12} & \cdots & p_{N}\end{array}\right]^{T}, \quad p_{i, j}(t)=1 / m_{i, j} \iint_{\Omega} \bar{q}$ $(x, y, t) W_{i, j}(x) \mathrm{d} x \mathrm{~d} y, \widetilde{M}_{w}, \widetilde{C}_{w}, \widetilde{K}_{w}$ are mass matrix, damping matrix, and stiffness matrix, respectively, in the form of

$$
\begin{aligned}
& \widetilde{M}_{w}=I, \\
& \widetilde{C}_{w}=c_{2} \widehat{\Psi}_{w}, \\
& \widetilde{K}_{w}=\omega^{2}+c_{1} \widehat{\Psi}_{w} \text {, }
\end{aligned}
$$

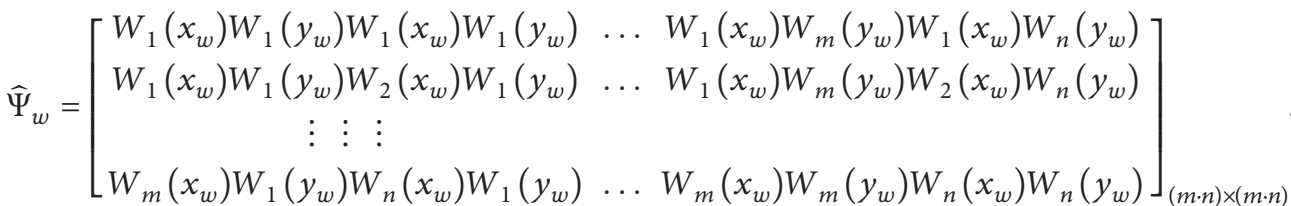

And it should be emphasized that in the later programming calculation, $c_{1}, c_{2}$ will also be matrices.

When there is no wave control force, the mass matrix $M$ is the unit matrix of $(m \cdot n) \times(m \cdot n), C$ is the zero matrix of $(m \cdot n) \times(m \cdot n)$, and $K$ is the diagonal matrix of the square of natural frequency. However, the wave control force couples the vibration modes of the uncontrolled original system together, and the modes of the wave control system change accordingly.

The state vector $X(t)=\left[q^{T}(t): \dot{q}^{T}(t)\right]^{T}$ is introduced, and equations (2a) $-(35)$ are written as

$$
\dot{X}(t)=A X(t)+B \tilde{p},
$$

where the coefficient matrix is

$$
\begin{aligned}
A & =\left[\begin{array}{cc}
0 & I \\
-\widetilde{M}_{w}{ }^{-1} \widetilde{K}_{w} & -\widetilde{M}_{w}{ }^{-1} \widetilde{C}_{w}
\end{array}\right], \\
B & =\left[\begin{array}{c}
0 \\
\tilde{M}_{w}{ }^{-1} \widehat{\Psi}_{d}
\end{array}\right], \\
\widehat{\Psi}_{d} & =\left[\begin{array}{ccc}
W_{1}\left(x_{1}\right) W_{1}\left(y_{1}\right) W_{1}\left(x_{2}\right) W_{1}\left(y_{2}\right) & \ldots & W_{1}\left(x_{N}\right) W_{1}\left(y_{N}\right) \\
W_{1}\left(x_{1}\right) W_{2}\left(y_{1}\right) & \ldots & W_{1}\left(x_{N}\right) W_{2}\left(y_{N}\right) \\
\vdots & \vdots & \vdots \\
W_{m}\left(x_{1}\right) W_{n}\left(y_{1}\right) & \ldots & W_{1}\left(x_{N}\right) W_{2}\left(y_{N}\right)
\end{array}\right]_{(m \cdot n) \times N}
\end{aligned}
$$

\section{Numerical Examples and Analysis Discussion}

The dynamic response of thin plates before and after applying wave control force is analyzed and studied by using feedback wave control. The characteristic length is taken as the length $b$ of the beam, and the following dimensionless quantity is adopted: Poisson ratio $v=0.30$; $a / b=2 ; \bar{\omega}_{i, j}=\omega_{i, j} / \omega_{1,1}(i=1,2,3 ; j=1,2)$. The first 6 dimensionless frequencies of the cantilever plate are shown in Table 1.

Figures 2-7 show the frequency response of the structure before and after applying wave control forces. In Figure 2, the unit disturbance force is applied at the point $\left(x_{d}, y_{d}\right)=(0.30 a, 0.20 b)$, while the wave control force and the measuring response position are at the point $\left(x_{w}, y_{w}\right)=$ $(0.40 a, 0.40 b)$ and $\left(x_{s}, y_{s}\right)=(0.40 a, 0.70 b)$, respectively. In Figure 3, the unit disturbance force is applied at the point $\left(x_{d}, y_{d}\right)=(0.40 a, 0.20 b)$, the wave control force and the measured response position are $\left(x_{w}, y_{w}\right)=(0.40 a, 0.40 b)$ and $\left(x_{s}, y_{s}\right)=(0.70 a, 0.80 b)$, respectively. The unit disturbance force is applied at the point $\left(x_{d}, y_{d}\right)=(0.30 a, 0.30 b)$ in Figure 4, the wave control force and the measured response position are $\left(x_{w}, y_{w}\right)=(0.45 a, 0.45 b)$ and $\left(x_{s}, y_{s}\right)=$ $(0.70 a, 0.60 b)$, respectively.

In Figure 5, the unit disturbance force is applied at the point $\left(x_{d}, y_{d}\right)=(0.40 a, 0.30 b)$, the wave control force and the measured response position are $\left(x_{w}, y_{w}\right)=(0.45 a$, $0.45 b)$ and $\left(x_{s}, y_{s}\right)=(0.40 a, 0.60 b)$, respectively. In Figures 6 and 7, the unit disturbance force is applied at the point $\left(x_{d}, y_{d}\right)=(0.30 a, 0.20 b)$, and the wave control force positions are $\left(x_{w}, y_{w}\right)=(0.40 a, 0.40 b), \quad\left(x_{w}, y_{w}\right)=(0.45 a$, $0.45 b)$, respectively, the measured response positions are $\left(x_{s}, y_{s}\right)=(0.40 a, 0.60 b)$ and $\left(x_{s}, y_{s}\right)=(0.40 a, 0.70 b)$, respectively. In the approximation of tuned PD control, the 
TABle 1: The first 6 dimensionless frequencies of the cantilever plate.

\begin{tabular}{lcccccc}
\hline Modal order & 1 & 2 & 3 & 4 & 5 & 6 \\
\hline Natural frequency & 1.00 & 51 & 5.51 & 12.90 & 15.43 & 23.73 \\
\hline
\end{tabular}

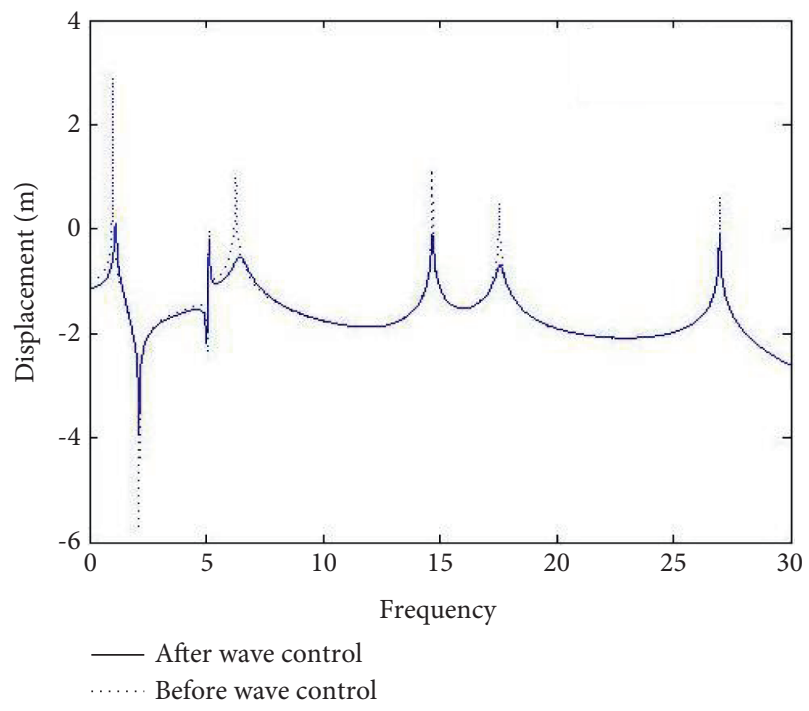

FIGURE 2: Frequency response before and after wave control.

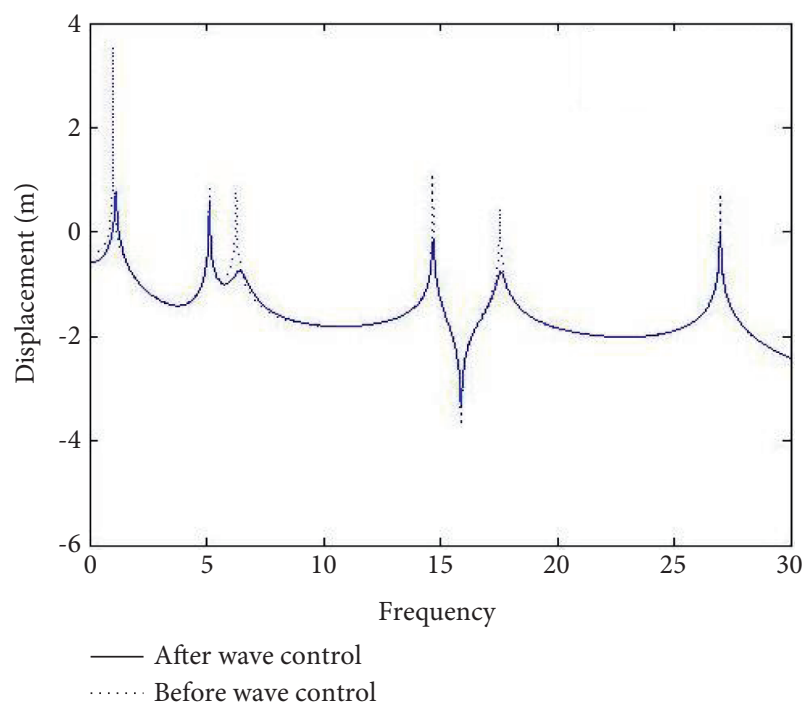

Figure 3: Frequency response before and after wave control.

controller is tuned to the optimum at the third natural frequency. Through analysis, the following are discussed:

It can be seen from Figures 2-7 that the frequency responses before and after different wave controls. The transverse coordinates are dimensionless frequency values, while the longitudinal coordinates are the common logarithmic values of frequency responses. Figures 2-7 show the frequency response of the front and rear structures using wave controllers. The response of the structure is rapid before control, while wave control can be regarded as adding damping to the structure and absorbing the energy carried

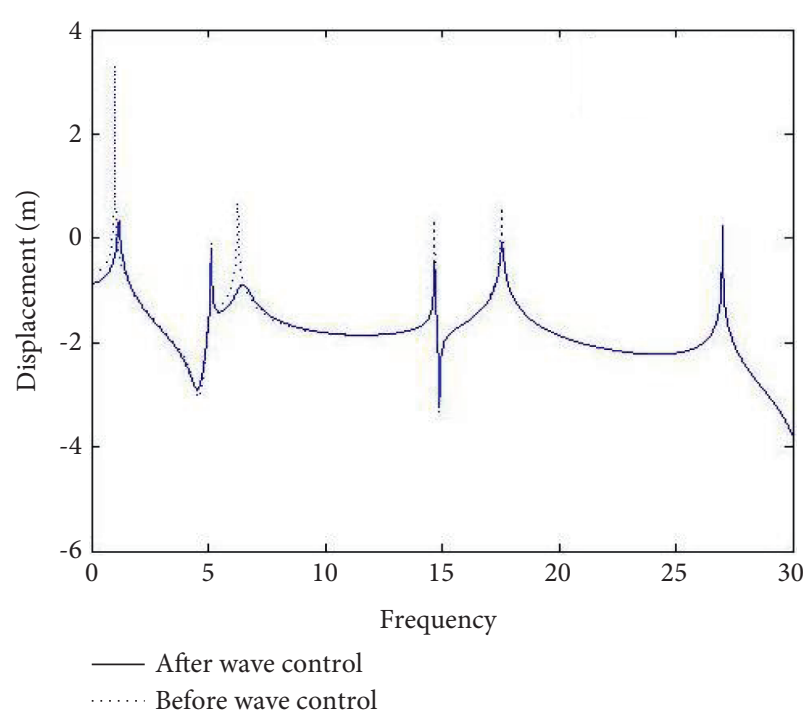

FIgURE 4: Frequency response before and after wave control.

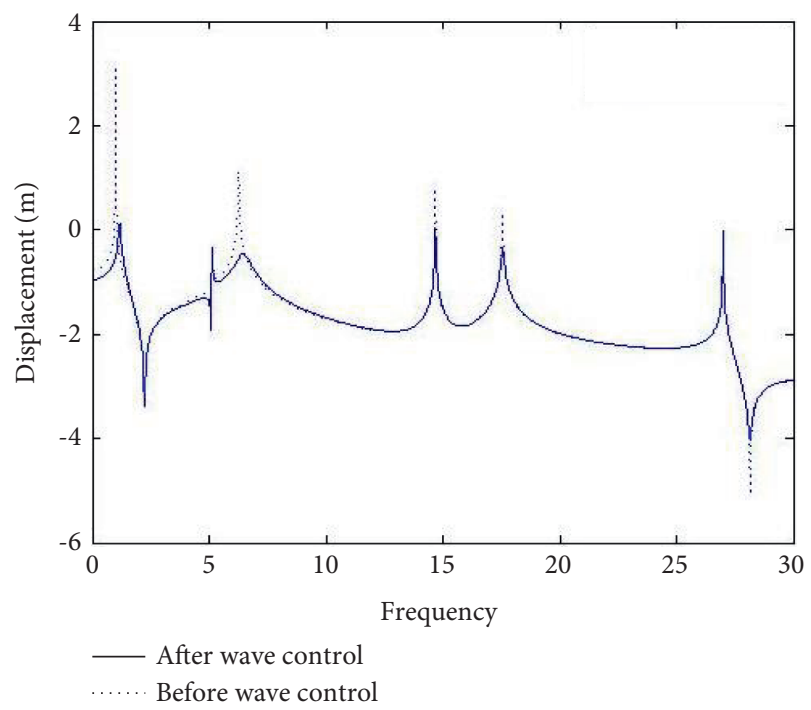

FIGURE 5: Frequency response before and after wave control.

by the elastic wave in the structure. After the wave control, the sharp response is weakened; in addition, the frequency response before and after the wave control also reflects that the modal characteristics of the whole structure have changed after the wave control, the natural frequency of the structure has been changed to a certain extent. Finally, from Figures 2-7, it can be seen that the position of the wave controller is different and the control effect of each order mode is different. At the third natural frequency, the absolute output amplitude of the controller is reduced to about $31.8 \%$ of the original value, and the controller is tuned to the optimum value at the third natural frequency, so the control effect is the best at the third natural frequency.

From the comparison of Figures 2-5, it can be seen that when the wave control position is the same, but the disturbance is different. And when the dynamic response position is different, although the sharp response is 


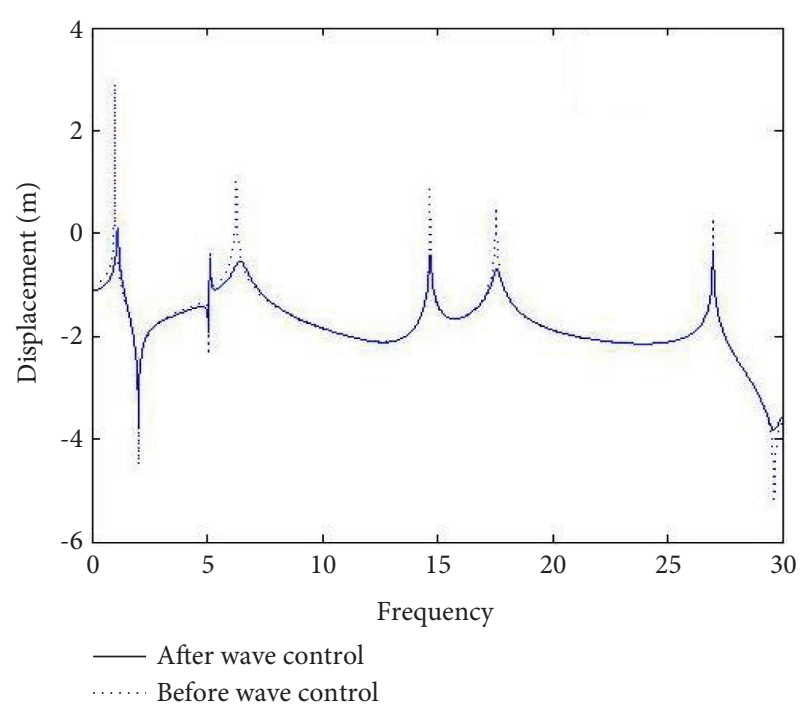

FIGURE 6: Frequency response before and after wave control.

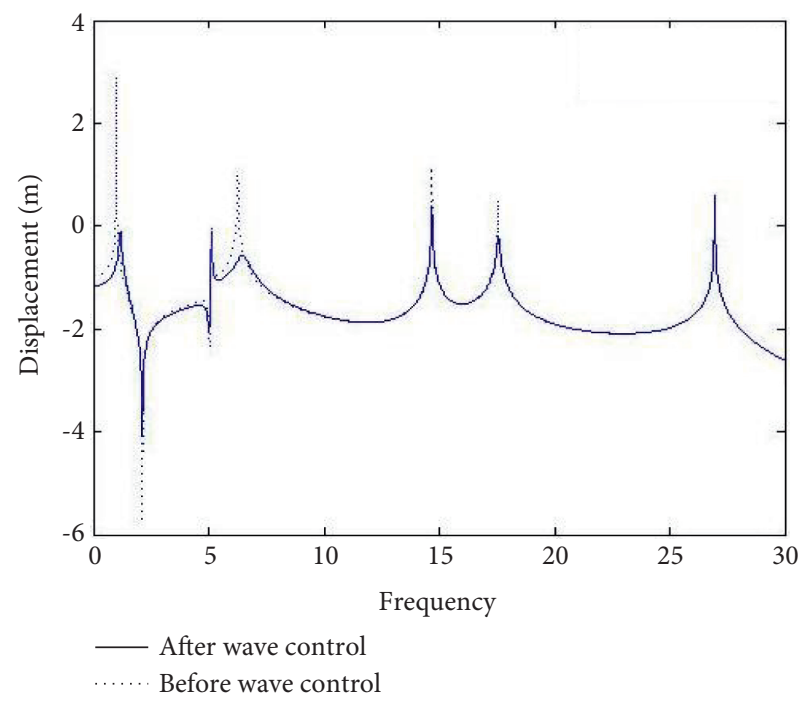

FIgURE 7: Frequency response before and after wave control.

weakened, the control effect is not identical. It can be seen from the comparison between Figures 6 and 7 that when the disturbance position is the same, the wave control force is different, and the dynamic response position is different, the control effect is also different. The influence of the application position of the wave controller on the control effect is also very important. The application point of the wave controller should avoid the node of the mode as far as possible. Otherwise, the active control will not get good results, and may even lead to system instability.

\section{Conclusions}

Based on the classical thin plate theory and the wave control method, the active vibration control of the cantilever plate is studied in this paper. In this paper, the wave control method widely used in the one-dimensional waveguide is applied to classical thin plates, and a series of active vibration control examples of cantilever plates are given. The following conclusions can be drawn:

The mode shape function of a cantilever rectangular plate is decomposed into the product of two functions, that is, the product of the mode shape function of a cantilever beam only dependent on the direction, and the mode shape function of a free beam at both ends only dependent on the direction. The problem of solving the vibration mode function of the thin plate is effectively solved.

When a series of wave control forces are applied to the plate, the point discontinuity on the beam is where the line discontinuity is. The wave control force is determined by using the equilibrium condition and continuity condition at the line discontinuity.

With wave control, a controller is designed to absorb the incident vibration energy by adding damping to the structure, which corresponds to a tuned spring and damper in the time domain. It can be seen from the control output that the control gain can achieve the optimal design at a certain tuning frequency. Therefore, when the applied position of wave control is different, the control effect of each order mode is different due to the distance between the applied position and the node of each order mode. For better control, the wave controller can be applied at several different locations, while avoiding instability of the system, the application point of the wave controller should avoid the modal nodes as far as possible.

\section{Data Availability}

The data used to support this study are available from the corresponding author upon reasonable request.

\section{Conflicts of Interest}

The authors declare that there are no conflicts of interest regarding the publication of this paper.

\section{Acknowledgments}

The project was supported by China's National Natural Science Foundation (Program No. 51275142).

\section{References}

[1] H. Moradi, M. Sadighi, and F. N. Bakhtiari, "Optimum design of a tuneable vibration absorber with variable position to suppress vibration of a cantilever plate," International Journal of Acoustics and Vibration, vol. 16, no. 2, pp. 55-63, 2011.

[2] M. Iqbal and F. U. Khan, "Hybrid vibration and wind energy harvesting using combined piezoelectric and electromagnetic conversion for bridge health monitoring applications," Energy Conversion and Management, vol. 172, pp. 611-618, 2018.

[3] A. Kodhanda, N. Ali, M. M. Sucheendran, and S. Talole, "Robust control of nonlinear resonance in a clamped rectangular plate," Journal of Vibration and Control, vol. 24, no. 18, pp. 4176-4194, 2018.

[4] J. Ma and H. J. Maris, "Localized vibrational modes in bars and plates," Journal of Applied Physics, vol. 107, no. 10, Article ID 104904, 2010. 
[5] B. Ni and C. Hu, "Dynamics of cantilever plates and its hybrid vibration control," Acta Mechanica Sinica, vol. 29, no. 5, pp. 738-748, 2013.

[6] A. S. M. Alzaidi, J. Kaplunov, and L. Prikazchikova, "Elastic bending wave on the edge of a semi-infinite plate reinforced by a strip plate," Mathematics and Mechanics of Solids, vol. 24, no. 10, pp. 3319-3330, 2019.

[7] C. R. Halkyard, "Sensor array design for wave decomposition in the presence of coupled motion," Journal of Sound and Vibration, vol. 259, no. 4, pp. 935-953, 2003.

[8] H. M. El-Khatib, B. R. Mace, and M. J. Brennan, "Suppression of bending waves in a beam using a tuned vibration absorber," Journal of Sound and Vibration, vol. 288, no. 4-5, pp. 1157$1175,2005$.

[9] A. A. Krushynska, "Flexural edge waves in semi-infinite elastic plates," Journal of Sound and Vibration, vol. 330, no. 9, pp. 1964-1976, 2011.

[10] I. S. Jones, N. V. Movchan, and A. B. Movchan, "Blockage and guiding of flexural waves in a semi-infinite double grating," Mathematical Methods in the Applied Sciences, vol. 40, no. 9, pp. 3265-3282, 2017.

[11] J. Kaplunov and A. Nobili, "The edge waves on a Kirchhoff plate bilaterally supported by a two-parameter elastic foundation," Journal of Vibration and Control, vol. 23, no. 12, pp. 2014-2022, 2017.

[12] S. H. Mirafza, A. M. Khorasani, and A. H. Ghasemi, "Optimizing time delay feedback for active vibration control of a cantilever beam using a genetic algorithm," Journal of Vibration and Control, vol. 22, no. 19, pp. 4047-4061, 2016.

[13] Z. Q. Jiang, C. Dou, and Y. X. Wu, "Experimental study on earthquake-resilient prefabricated cross joints with L-shaped plates," Engineering Structures, vol. 184, pp. 74-84, 2019.

[14] B. Ni and C. Hu, "Dynamics of the Mindlin plate and its modal vibration control," Journal of Vibration and Control, vol. 18, no. 13, pp. 2039-2049, 2012.

[15] T. Chen, C. Hu, and W. H. Huang, "Vibration control of cantilevered Mindlin-type plates," Journal of Sound and Vibration, vol. 320, no. 1-2, pp. 221-234, 2009.

[16] J. S. Bae, J. S. Park, J. H. wang, and J. H. Roh, "Vibration Suppression of a Cantilever Plate Using Magnetically Multimode Tuned Mass Dampers," Shock and Vibration, vol. 2018, Article ID 3463528, 2018.

[17] Y. Cao, M. M. Li, and Z. Z. Gao, "Dynamic testing and analysis of Poisson's ratio of lumbers based on the cantilever-plate bending mode shape method," Journal of Testing and Evaluation, vol. 47, no. 4, pp. 2540-2550, 2019.

[18] E. Omidi and S. N. Mahmoodi, "Vibration suppression of distributed parameter flexible structures by Integral Consensus Control," Journal of Sound and Vibration, vol. 364, pp. 1-13, 2016.

[19] Z. C. Qiu, H. X. Wu, and D. Zhang, "Experimental researches on sliding mode active vibration control of flexible piezoelectric cantilever plate integrated gyroscope," Thin-Walled Structures, vol. 47, no. 8-9, pp. 836-846, 2009.

[20] R. Li, P. C. Wang, and L. H. Tong, "On new analytic free vibration solutions of rectangular thin cantilever plates in the symplectic space," Applied Mathematical Modelling, vol. 53, pp. 310-318, 2018.

[21] Z. C. Qiu, T. X. Wang, and X. M. Zhang, "Sliding mode predictive vibration control of a piezoelectric flexible plate," Journal of Intelligent Material Systems and Structures, vol. 21, no. 1, pp. 65-81, 2021.

[22] P. Khandagale, G. Bhakar, and S. S. Joshi, "Modelling timedomain vibratory deflection response of thin-walled cantilever workpieces during flank milling," Journal of Manufacturing Processes, vol. 33, pp. 278-290, 2018.

[23] P. X. Sun, H. Yang, and Y. Zhao, "Time-domain calculation method of improved hysteretic damped system based on frequency-dependent loss factor," Journal of Sound and Vibration, vol. 488, Article ID 115658, 2020.

[24] H. J. Kim, "A study on the design of a double cantilever structure friction tester for precision friction measurement," Tribology and Lubricants, vol. 34, no. 4, pp. 125-131, 2018.

[25] I. Chenini, R. Nasri, C. Mrad, and Y. Abdelli, "Kinematics effect on honeycomb sandwich beams vibration," Mechanics \& Industry, vol. 18, no. 3, Article ID 2016039, 2017. 